\title{
Why children are less affected by COVID-19 than adults: potential immunological factors and the renin-angiotensin system associated mechanisms
}

\section{Krogh-Jensen O.A. ${ }^{1,2}$, Nikitina I.V. ${ }^{1}$, Donnikov A.E. ${ }^{1}$, Lenyushkina A.A. ${ }^{1}$, Degtyareva N.D. ${ }^{2}$, Degtyareva A.V.1,2}

1) National Medical Research Center for Obstetrics, Gynecology, and Perinatology named after Academician V.I. Kulakov of the Ministry of Health of the Russian Federation; Department of pediatrics and neonatology; Oparina str. 4, Moscow, Russia

2) Federal State Autonomous Educational Institution of Higher Education I.M. Sechenov First Moscow State Medical University of the Ministry of Health of the Russian Federation (Sechenov University); Pediatric faculty, neonatal department; 8/2 Trubetskaya str., Moscow, Russia

*Contributed equally

Corresponding author: Olga Krogh-Jensen, MD; http://orcid.org/0000-0002-5178-5659

NICU of the National Medical Research Center for Obstetrics, Gynecology and Perinatology named after Academician V.I. Kulakov of the Ministry of Health of the Russian Federation; Department of pediatrics and neonatology; Oparina str. 4, Moscow, Russia.

E-mail: o_krogh@oparina4.ru; olgaborisevich@gmail.com

Phone: +79260140135

There's no conflict of interest

Abstract. The spread of the infection caused by the new coronavirus SARS-CoV-2 (COVID-19) became pandemic on March 11, 2020. From the time of the first cases (in November 2019, Wuhan, China), to date, a large number of COVID-19 observations have been accumulated in different age groups of patients both in China and abroad. Published scientific data allows us to conclude that children suffer from COVID-19 much less often than adults and tolerate the disease in a milder form, often appear to be asymptomatic. There is currently no final answer why children are less susceptible to this virus; however, scientists are increasingly inclined to consider a complex effect of the immune response and components of the renin-angiotensin system (RAS), which according to recent studies affects not only the cardiovascular system, but is also responsible for the activation of inflammatory reactions. A hypothesis of genetic predisposition to the development of severe forms of COVID-19 has recently been made. 
We conducted a search for publications in the databases and showed current scientific ideas about COVID-19 pathogenesis and factors influencing the disease development in childhood.

Childhood immunity may have several protective features against SARS-CoV-2: immaturity of particular elements of the innate immune response, constitutional lymphocytosis with a shift towards anti-inflammatory Th2-response, as well as "trained" immunity.

The influence of renin-angiotensin system reactions in this review is shown from two perspectives: expression of ACE2 receptors and polymorphisms of certain genes of this system.

It was established that ACE2 transmembrane protein is not only the entry point for the virus but also plays a regulatory role, turning the pro-inflammatory vasoconstrictor angiotensin II into antiinflammatory angiotensin (1-7), which has vasodilating properties. Higher ACE2 content in children compared with adults helps maintain balance in the renin-angiotensin system and prevents the development of complications. It was also shown that the presence of certain genetic polymorphisms (AGTR1, AGTR2, ACE2, ACE) could determine the imbalance inside the RAS, leading to more pronounced reactions of alveolocytes, vascular endothelium and smooth muscle fibers in response to SARS-CoV-2 infection due to a shift towards vasoconstrictor, proliferative and profibrotic mechanisms.

Keywords: SARS-CoV-2; COVID-19; pathogenesis; children; neonates; immune response; secondary hemophagocytic lymphohistiocytosis; renin-angiotensin system; genetic polymorphisms; ACE2; AGTR2; AGTR1; NOS; lung injury.

\section{List of abbreviations:}

ACE - angiotensin converting enzyme

ACE2- angiotensin converting enzyme 2

Ang-I - angiotensin I

Ang-II - angiotensin II

Ang-(1-7) - angiotensin (1-7)

Ang- (1-9) - angiotensin (1-9)

AGTR1 (AT1) - Angiotensin II Receptor Type 1

AGTR2 (AT2) - Angiotensin II Receptor Type 2

ARDS - acute respiratory distress syndrome;

BAL - bronchoalveolar lavage; 
COVID-19 - COronaVIrus Disease 2019

GA - gestational age

IFN $\gamma$ - interferone $\gamma$

$\mathrm{IRF}$ - interferon regulatory factors

MAS - MAS-receptor for Angiotensin (1-7)

MERS - Middle East Respiratory Syndrome

NK- natural killers

NF- $\kappa \mathrm{B}$ - nuclear factor kappa-light-chain-enhancer of activated B cells

RAS - renin-angiotensin system

RDS - respiratory distress-syndrome

sHLH - secondary hemophagocytic lymphohistiocytosis

Th1 - T-helper type 1

Th2 - T-helper type 2

TLR - Toll-like receptors

TMPRSS2 - Transmembrane Serine Protease 2

TNF- $\alpha-$ Tumor necrosis factor alpha

TTN - transient tachypnea of the newborn

\section{Introduction}

Coronaviruses (subfamily Orthocoronavirinae) belong to the family Coronaviridae and represent a group of enveloped single-strained RNA viruses, including four genera (alpha, beta, gamma, delta) and about 40 species. The first human coronavirus was discovered in the 1960s by Tyrrell and Bynoe and, currently, there are seven coronaviruses that are pathogenic for humans: 2 of which belong to the alpha-coronaviruses (hCoV E229 and hCoV NL63) and 5 - to the betacoronaviruses (hCoV OC43, hCoV HKUI, SARS-CoV, MERS-CoV and the new SARS- CoV 2). Over the past decades, two outbreaks of acute respiratory distress syndrome (ARDS) and pneumonia caused by coronaviruses have already been reported (SARS-CoV in 2003 and MERS$\mathrm{CoV}$ in 2013). However, only the spread of the new coronavirus SARS-CoV-2, causing the “Coronavirus disease 2019” (COVID-19), became pandemic (on March 11, 2020).

From the onset of the first COVID-19 cases (in November 2019, Wuhan, China), to date, a large number of observations of the course of the disease have been accumulated in different age groups of patients both in China and abroad. Published scientific data allows us to conclude that 
children suffer from COVID-19 much less often than adults and tolerate the disease in a milder form, often appear to be asymptomatic. According to Chinese researchers, children 0-9 years old make up $0.9 \%$ of all confirmed cases; children $10-19$ years old account for $1.2 \%$ of the number of cases ${ }^{1,2}$. Similar results are shown by the National Italian report dated $23.03 .20(318(0.5 \%)$ confirmed cases in the age group 0-9 years, and $386(0.7 \%)$ confirmed cases in the age group 1019 years) and the US morbidity and mortality report as of 2.04.20 (the age group of 0-18 years is $1.7 \%$ of all infected $)^{3}$. The incidence of COVID-19 severe forms in children and the need for intensive care is also significantly lower than that in adults. Children account for $0.25 \%$ of the total number of patients admitted to the intensive care units in Italy (the Lombardy region) ${ }^{4}$. Reports on neonatal COVID-19 cases throughout the World remain rare ${ }^{5-7}$.

Given that usually, respiratory viral infections dominate in children, experts were baffled over their low susceptibility to the pathological effects of SARS-CoV-2. There is currently no definitive answer to this; however, scientists consider the impact of age-dependent immune response features along with the influence of renin-angiotensin system (RAS), which, according to recent studies besides cardiovascular regulation, takes part in the activation of inflammatory reactions ${ }^{8,9}$.

Genetic predisposition to the development of severe forms of COVID-19 has been little studied. However, genetic trials of lung damage in other serious respiratory diseases such as cystic fibrosis, neonatal pneumonia, bronchopulmonary dysplasia, have revealed genes that regulate the main pathological processes in the lung tissue ${ }^{10}$. A statistically significant relationship has been shown between the severity of the disease and certain allelic variants of the genes of innate immunity, regulators of vascular tone, and energy metabolism, including the RAS system, which could be considered in the future as possible therapeutic targets.

The purpose of this publication is to review current scientific ideas about COVID-19 pathogenesis and analyze factors that influence disease development in childhood. We conducted a search for publications in the databases PubMed, ClinicalKey, MedLine, Google scholar, dealing with characteristics of SARS-CoV-2, COVID-19 in children, ACE2, renin-angiotensin system, genetic polymorphisms and their relationship with infections and lung damage.

\section{Immunological aspects of COVID-19 course}

\section{Structural Features of SARS-CoV-2}

SARS-CoV-2 differs from all other common respiratory viruses. Various viruses use different receptors, both for penetration into host cells and for replication followed by virions' 
assembly. The heterogeneity of the receptors and their distribution determines primary localization and severity of the infectious process. So, for example, the alpha-coronavirus hCoV-229E uses human aminopeptidase $\mathrm{N}$ (human aminopeptidase N - hAPN) for its endocytosis, which causes nasal epithelium damage. The Influenza virus, in its turn, penetrates through sialylated glycoconjugates on the surface of the laryngeal and tracheal epithelium, and on condition of further spread it causes diffuse inflammation in the bronchi and bronchioles ${ }^{11,12}$. The Penetration of SARS-CoV-2 in the cell occurs via surface S-spike proteins, which are distinctive for betacoronaviruses. S-spikes of SARS-CoV-2 interact with protease activity region of angiotensinconverting enzyme 2 (ACE2) that is expressed in different human tissues: nasal and pharyngeal epithelium, on membranes of pneumocytes type 2, enterocytes, endothelial cells, as well as on the surface of smooth muscle cells in most organs ${ }^{13}$. The viral S-spike is activated by the membranebound serine protease 2 (TMPRSS2), which also cleaves ACE2 and facilitates viral fusion with the host cell membranes ${ }^{14}$. Another target for SARS-CoV-2 on the cell surface is CD147 protein, which is used not only by SARS-CoV and SARS-CoV-2 but also by malarial plasmodium ${ }^{15}$. The features mentioned above lead to a fundamentally different organism response to SARS-CoV-2 compared to other widespread respiratory viruses. This response consists of the complex activation of both the immune response and the renin-angiotensin system, which is currently under active investigation ${ }^{16,17}$.

\section{Current views on the pathogenesis of COVID-19}

SARS-CoV-2 binds to receptors on the surface of the epithelium of the upper and (or) lower respiratory tract, penetrates the cells, binds there with endosomal Toll-like receptors (TLRs) 3 and 7, and cytoplasmic RNA receptors. This launches a cascade of innate immunity reactions: activation of $\mathrm{NF}_{\mathrm{K}} \mathrm{B}$ and IRF pathways, production of interferons, interleukin 1, and activation of adaptive cellular immunity. The predominant role in the adaptive immune response is played by the adequate activity of CD8 + cytotoxic T-lymphocytes, which provide the release of cytotoxic granules and interferons and CD4 + T-helpers, that trigger humoral immunity. With a favorable course of the disease, the antiviral activity of the body thus is being formed, and it restricts the excess inflammation, terminates virus replication, provides its elimination. Effective antiviral immune response, in most cases, apparently doesn't lead to the lower respiratory tract damage ${ }^{18,19}$.

A feature of SARS-CoV-2 is its ability in some cases to "bypass" the effective antiviral immune response at the level of innate and local mechanisms and to launch a cascade of reactions 
of severe, persistent inflammation. In this case, excessive release of pro-inflammatory cytokines along with early production of immature/ineffective antibodies followed by the formation of immune complexes leads to continued virus replication in antigen-presenting cells, that aggravate inflammatory response causing various tissues damage with immune complexes.

In parallel with the initiation of immune responses, SARS-CoV-2 inhibits ACE2 activity, leads to an imbalance in the renin-angiotensin system (RAS), a lack of angiotensin (1-7) and excessive accumulation of angiotensin II (Ang-II), which is a potent vasoconstrictor and has a proinflammatory, profibrotic and prothrombotic effect ${ }^{20}$. The cascade of RAS reactions is described in detail below. The current conception of the pathogenetic mechanisms of COVID-19 is presented in Fig.1.

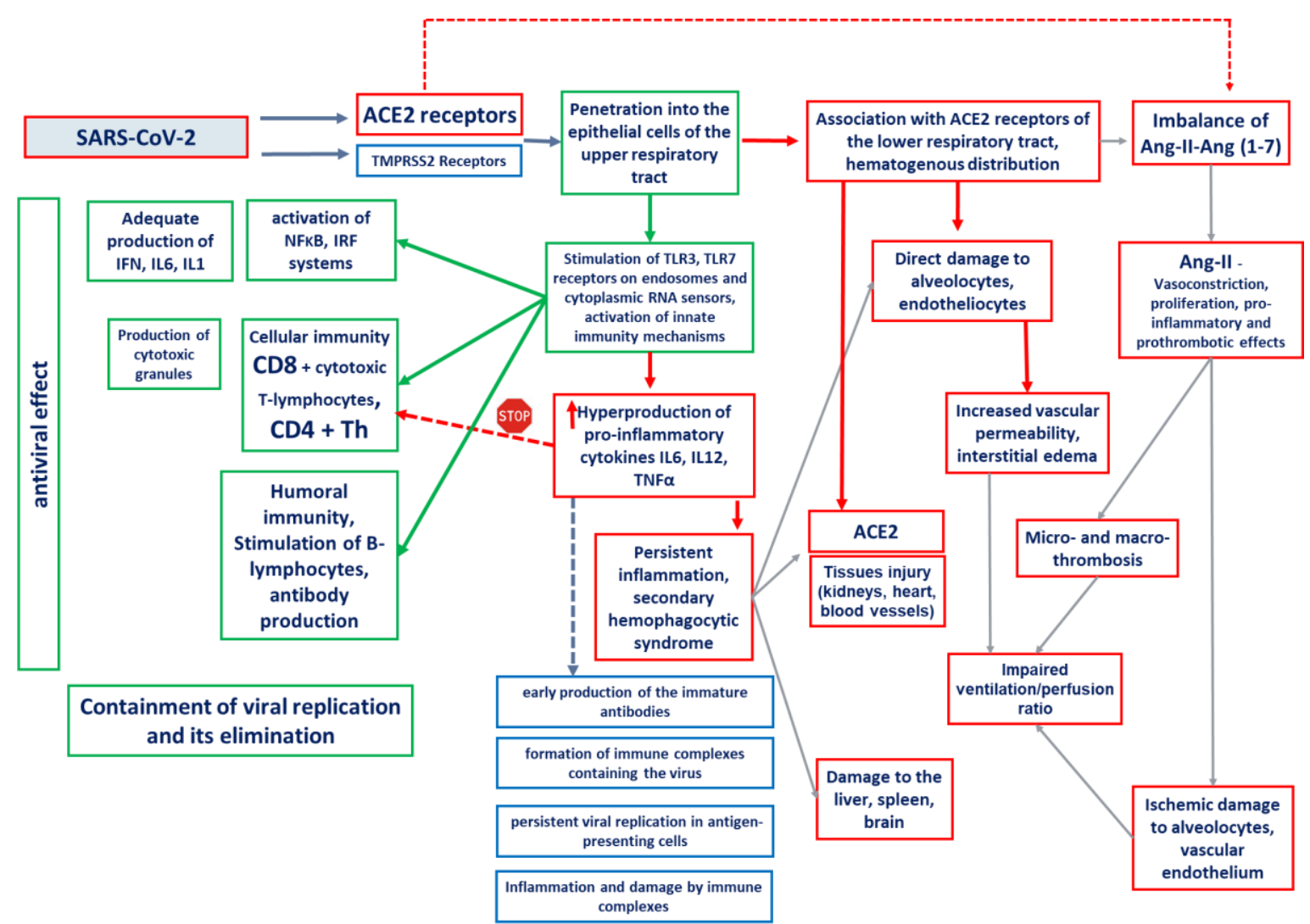

Figure 1. The current conception of COVID-19 pathogenesis

ACE2- angiotensin converting enzyme 2

TMPRSS2 -Transmembrane Serine Protease 2

This response of the immune system is very similar to that in patients suffering from diseases in which secondary hemophagocytic lymphohistiocytosis (sHLH) develops, in the literature it is often called secondary hemophagocytosis ${ }^{21-25}$. This syndrome is an acquired 
immune system dysfunction that develops in patients with autoimmune diseases, severe infections (especially viral etiology), some tumors. sHLH is a life-threatening condition, and its pathophysiological substrate lies in the excessive proliferation of activated T-cells and macrophages that accumulate in the liver, spleen, and lymph nodes. Systemic macrophage activation, hyperproduction of pro-inflammatory cytokines, and hypercytokinemia underlie cytotoxic effects (Fig. 2). A distinctive, but non-specific morphological sign of the syndrome is hemophagocytosis. Systemic activation of macrophages leads to the initiation of spontaneous phagocytosis of the blood cells (platelets, red blood cells, and polymorphonuclear cells) ${ }^{26}$.

Tissue infiltration with activated CD8 + T-lymphocytes and macrophages in combination with hypercytokinemia are classic features of sHLH, that leads to the cytopenia development, hemorrhagic syndrome, coagulopathy, extensive tissue necrosis and the development of multiple organ failure. Previously, sHLH was usually considered in relation to a narrow circle of patients with rheumatological and onco-hematological profiles. However, nowadays, thanks to an understanding of the pathogenetic mechanisms of sHLH, it is regarded as a universal clinical and pathological phenomenon, the development of which can occur in severe cases of a wide range of diseases of various etiologies, gradually expanding the issue to the intensive care area.

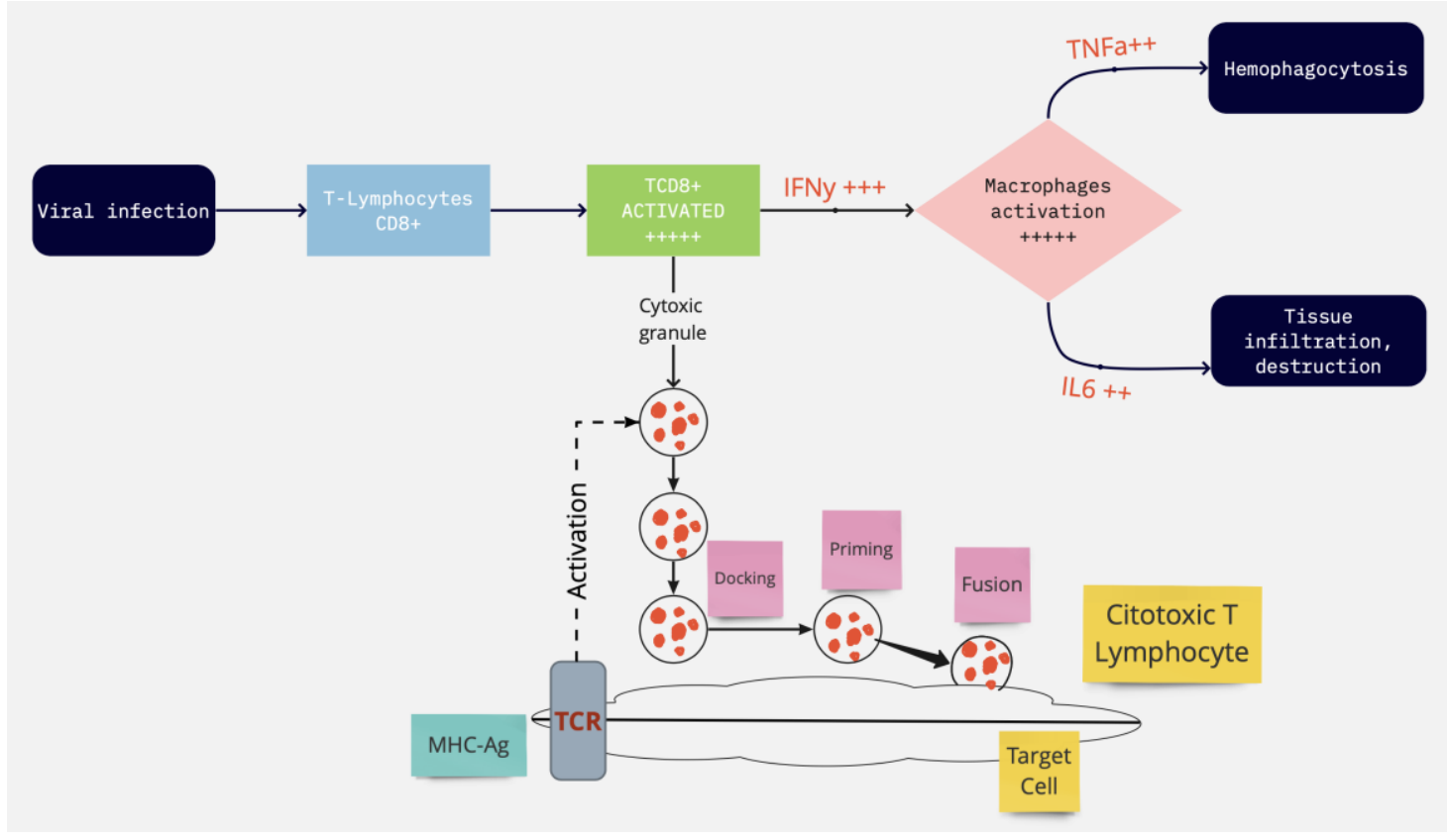

Figure2. Mechanisms leading to the occurrence of hemophagocytic syndrome.

MHC-Ag, Major histocompatibility complex antigen

TCR, T-cell receptor 


\section{Protective immunological mechanisms}

Currently, most authors tend to believe that children's resistance to COVID-19 is determined by a number of characteristics of the immune system.

The development of the human immune system is a continuous process, starting from the embryonic period and ending with the period of adulthood. Normally, intrauterine development of the fetus occurs under conditions of low antigenic stimulation, so the immune system of healthy full-term newborns is vastly different from the immune system of adults. During a normal pregnancy, the fetal immune system and the mother's immune system are in a state of equilibrium and immunological tolerance. The neonatal immune response is aimed to suppress the development of the Th1-type response and the prevalence of the Th2-type response, which ensures the maintenance of tolerance to maternal antigens in utero. "Immunological provocation" by the fetus in relation to the mother's body can lead to premature birth. In this connection, the normal immune response of newborns is biased towards the anti-inflammatory Th2 response.

In comparison with adults, children (newborns, in particular) have immature innate immunity response. It is acknowledged that induced neonatal mononuclear cells produce significantly less Th1 proinflammatory cytokines (TNF- $\alpha$, IFN $\gamma$ ) as compared with adult ones ${ }^{\mathbf{2 7}-}$ 30. Against the backdrop of unformed adaptive immunity, the primary importance of protecting newborns from infection belongs to phagocytes, that rapidly migrate to the pathogen invasion site, recognize and neutralize it due to bactericidal enzymes, and non-protein molecules' effects; as well as to neutrophilic granulocytes and monocytes that have an immunoregulatory effect ${ }^{\mathbf{3 1}, \mathbf{3 2}}$. As a result of these specifics, infectious process generalization in newborns is seen more often than in older children and adults in most infections except for COVID-19 ${ }^{\mathbf{3 3}}$.

Inflammation as an evolutionarily developed protective process, but at the same time, it may have damaging effects on the body. Secondary alteration is tissue damage that does not depend on the direct etiological factor effect and can continue even when this agent is no longer existing. The etiological factor is the trigger mechanism of the process; after it, the inflammation will proceed according to the tissue and organ specifics. Therefore, when the body comes into contact with an infectious agent in response to its toxins and waste products, both proinflammatory and anti-inflammatory cytokines are simultaneously produced. They control and limit the inflammatory process to minimize the damage. A cytokine storm (cytokine cascade, 
hypercytokinemia) is an uncontrolled inflammation that leads to the damage of the body's own tissues. This, in particular, explains the large number of cases during the "Spanish flu" epidemic (the influenza pandemic of 1918) among young, healthy people as the most vulnerable cohort due to high immunoreactivity.

The second immunological factor that can favorably influence the course of the disease is the cellular composition of the blood. Compared with adults, the absolute number of lymphocytes in children and newborns is higher, and the Th2 type prevails in the population ${ }^{30}$.

Some experts assume that "trained immunity" may be linked to a low frequency of COVID19 in children. The concept of this phenomenon was proposed not so long ago, in 2013 by Netea M.G. It consists of the long-term functional reprogramming of innate immune cells, which is induced by exogenous or endogenous stimuli, causing a persistent increase in innate immune defense against a wide range of infectious agents. It is caused by preliminary contacts with pathogens, and being more precisely, with their molecular patterns. In children of preschool and school-age, unlike adults, there is a so-called constant training of the immune system in connection with frequent viral infections, routine vaccination, and an extended stay in children's groups. That

causes fast and effective host protective response when exposed to various pathogens ${ }^{9,34}$. The concept of "trained immunity" has also been actively discussed by some authors, assuming the existing association of a low incidence of severe cases of COVID-19 and vaccination against tuberculosis with BCG ${ }^{35,36}$.

Thus, based on scientific data to date, we can conclude that immature innate immunity response, a constitutionally high level of lymphocytes with a shift towards Th-2 type in their population, and the bias towards anti-inflammatory immunological response constitute a kind of defense against COVID-19. This protection is also due to the low level of stimulatory activity of Th- 1 cells, monocytes and dendritic cells, as well as active "trained immunity" in preschool and school children, which provides active antiviral protection without developing an excessively intense cascade of immunological reactions.

\section{Studies of the renin-angiotensin system}

1. The role of ACE2 in the pathogenesis of COVID-19. Age and gender differences in ACE2 expression as a factor associated with the disease severity. 
Speaking of the disease inception and the launch of immune reactions, a lot of attention is currently paid to the role of angiotensin-converting enzyme 2 (ACE2 - angiotensin-converting enzyme 2), a membrane protein and one of the components of the renin-angiotensin system (RAS). ACE2 is widely present on the membranes of cells of the upper respiratory tract, lungs, heart, kidneys, and intestinal wall. This protein catalyzes the conversion of Angiotensin II to Angiotensin (1-7) (Fig. 3). The role of RAS cascade has been extensively studied in the regulation of blood pressure, renal and cardiovascular activity, but it is now recognized that RAS has several functions in the body, including lung processes ${ }^{17,37,38}$.

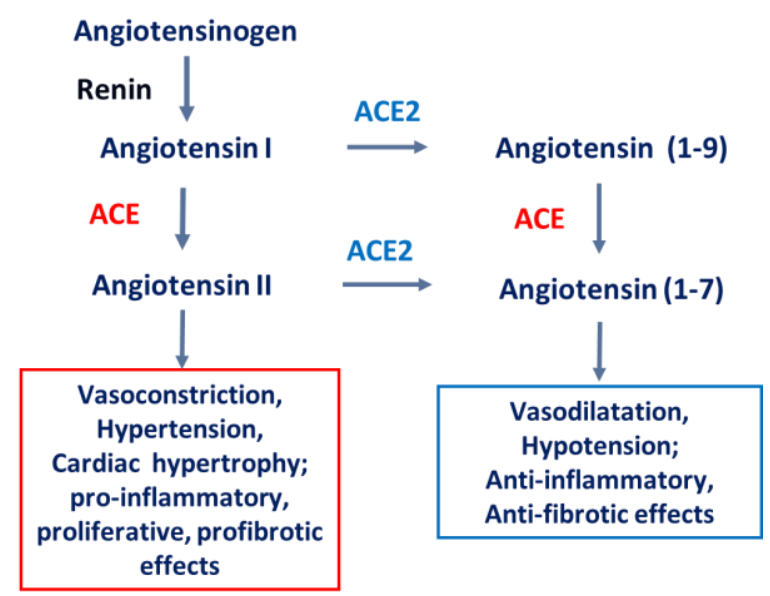

Figure 3. Contemporary view on the renin-angiotensin system

It is necessary to mention that Angiotensin II (Ang-II), one of the main hormones of RAS, induces apoptosis of endothelial cells in pulmonary arteries and alveolar epithelial cells, plays an important role in the fibrous response to acute lung damage (causing transformation of the growth factor $-\alpha$ expression in the lungs, promotes the proliferation of fibroblasts), triggers hypertrophic and hyperplastic growth of vascular smooth muscle cells (myointimal hyperplasia) and regulates the expression of NO synthetase ${ }^{39,40}$. Also, Angiotensin II in vitro proved to be an activator of inflammation, increasing the synthesis of pro-inflammatory cytokines and chemokines through the AT1 and AT2 receptors, followed by activation of the NF- $\kappa$ B pathway (nuclear factor kappa B). $\mathrm{NF}-\kappa \mathrm{B}$ is a protein complex that regulates DNA transcription, cytokine production, and cell survival. NF- $\kappa \mathrm{B}$ plays a key role in regulating the immune response to infection. Angiotensin (17), in its turn, has the opposite, protective, properties - vasodilation, antiproliferative effect. Excessive accumulation of Ang-II and bradykinin may cause interstitial pulmonary edema, acute respiratory distress syndrome and myocarditis. 
The association of viral invasion with ACE2 receptors was detected during previous epidemics of the coronaviruses SARS (2002) and MERS (2013). Since the amino acid sequence of the S-spike (S-protein) on the surface of the SARS-CoV-2 virus repeats the structure of that of other coronaviruses by $76-80 \%{ }^{41}$, by the time of the outbreak of a new coronavirus infection, the features of the interaction of the virus with ACE2 were already well studied.

After the high affinity connection with host ACE2, the virus fulfills fusion with host cell and penetration into it. Currently, there are 2 main (inherently opposite) theories about the role of ACE2 in the development of COVID-19 disease. According to the first hypothesis, ACE2 play a protective role. After penetration into the cell, the virus suppresses ACE2 activity and accordingly shifts the balance in the renin-angiotensin system towards the accumulation of pro-inflammatory Ang-II. Therefore, the presence of ACE2 in large quantities on the cell membranes, as well as the high concentration of free ACE2, contributes to the protective effect of Angiotensin (1-7), which prevents the development of uncontrolled inflammation and vasoconstriction.

According to the second hypothesis, ACE2 is considered as the main point of virus entry and, therefore, the greater the expression of this protein on cell membranes is, the higher the likelihood of the disease. Current understanding of the relationship between COVID-19 and RAS is shown in Figure 4.

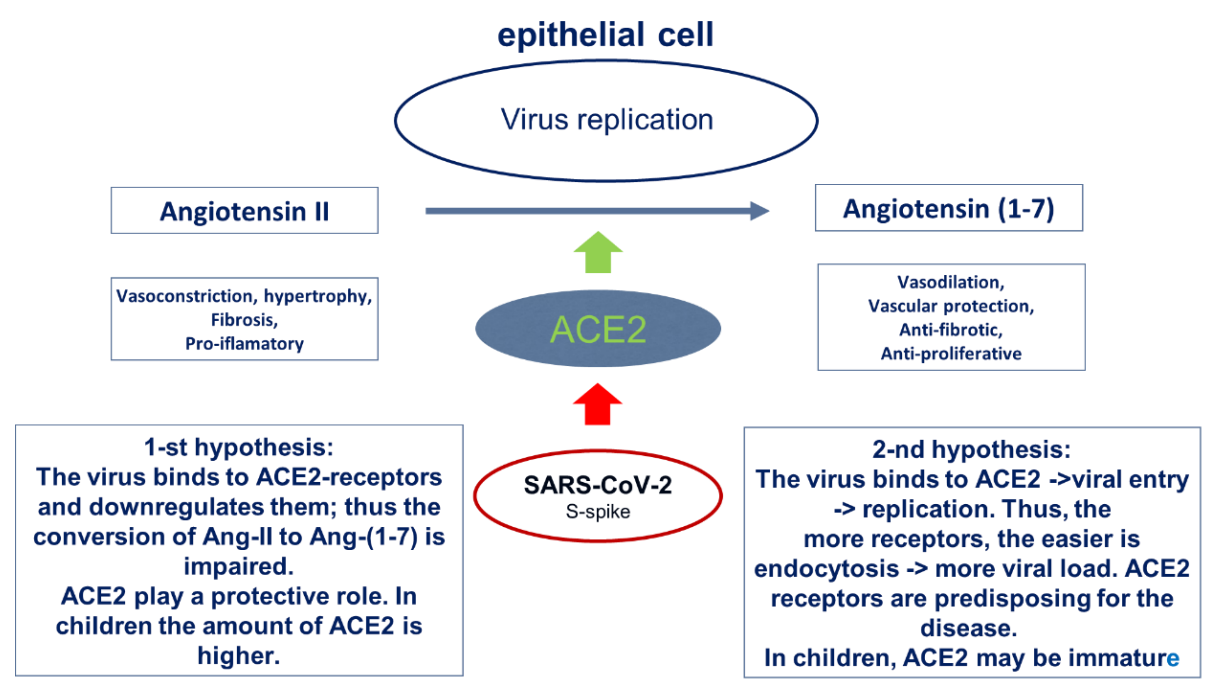

Figure 4. Current understanding of the relationship between COVID-19 and RAS

The first hypothesis is confirmed by a number of studies of the previous decade, which are mainly carried out on animals. So Xie et al. in their laboratory work on rats showed that ACE2 expression on the epithelial membranes of the bronchi, alveoli, endothelium and smooth muscle 
cells of the pulmonary vessels decreases significantly with age; the dependence of ACE2 content on sex was also observed in older individuals (in females is higher than in males) ${ }^{42}$. Dutch scientists investigated the content of RAS components in broncho-alveolar lavage (BAL) in rats with artificially induced acute induced respiratory distress syndrome (ARDS). A noticeable decrease in ACE2 was detected in rats undergoing mechanical ventilation, which contrasted with the increased content of free (soluble) ACE2 protein in these samples, which was observed using Western blotting. Immunohistochemical studies have shown that there is apparently local RAS in the lungs: lung fibroblasts, alveolar macrophages and epithelial cells are able to express genes encoding RAS components and synthesizing RAS peptides such as ACE and ACE2, as well as AT1 receptors and MAS-receptors. It was found that ACE and ACE2 are the main enzymes that control local amounts of Ang-II and Ang (1-7) ${ }^{43}$.

ACE2's protective role was shown by Kuba et al. during the previous SARS-CoV epidemic 44 . These results were confirmed by Chen during the current COVID-19 pandemic: ACE2 expression was higher in women than in men and decreased with age, regardless of gender; it was also found that estrogens and cytokines are involved in ACE2 expression ${ }^{45}$. However, the methodology of this study, described in the preliminary version of the article, is poorly defined and does not allow the conclusions to be drawn, especially regarding the children's population.

The findings of age- and sex-related ACE2-expression are matching with actual COVID19 statistics: less severe disease course in women. In Spain, men and women comprise about an equal number of Covid-19 cases, as the country's Health Ministry reported on April 3, $2020{ }^{46}$. But men were more than twice as likely to wind up in the ICU — and more likely to die making up to almost two-thirds of deaths. Analysis of data from about 2,000 patients during China's main outbreak, from December through February, showed that about 60 percent of patients were men. And China's Center for Disease Control reported a fatality rate of 2.8 percent for men versus 1.7 percent for women ${ }^{2}$.

There is currently insufficient evidence to support the second theory about the negative effects of the high ACE2 content. The studies were conducted on small and heterogeneous samples. Repeated citation of very dubious studies published during the pandemic in the form of reprints that did not pass a detailed expert analysis is also noted ${ }^{1}$.

Looking at the results of recent decades studies as a whole, we can conclude that the elements of the RAS are widely represented in the lungs. In addition to the main, vasoregulatory function, they are actively involved in the process of inflammation. It was found that Ang-I and 
Ang-II are potent inflammation activators, which promote tissue damage via interaction with the receptors AGTR1 and AGTR2 on alveolocytes and endotheliocytes. The subsequent development of interstitial edema and infiltration, together with the strong proliferative and profibrotic effect of Ang-II, eventually leads to fibrosis. The degree of aggressiveness of Ang-I and Ang-II effects is determined by the amount of the enzyme ACE2, which decreases their level, turning them into Ang (1-7). In this regard, a higher ACE2 content in children compared to adults prevents imbalance in the RAS and, respectively, protects cells and tissues from the damaging effects of Ang-II.

2. Genetically determined features of pro- and anti-inflammatory elements balance inside the RAS system (Ang-II - Ang (1-7)) as a factor that can determine the severity of the COVID-19 course and possible complications.

We suggest that the balance between pro- and anti-inflammatory components of the RAS is determined not only by age and gender characteristics of ACE2 expression but also by genetic mechanisms. Genetic studies of the RAS have shown that there is a relationship between not only one but several genes of this system with the development of severe pulmonary pathology and cardiovascular system.

There are currently no convincing data on the isolated effect of ACE2 polymorphism on the incidence and severity of the COVID-19. A large-scale population-based study conducted in the Netherlands did not find a statistically significant relationship between this gene and lung, heart, or kidney damage, despite a large sample (blood samples of 36,339 people collected by Lifelines biobank in 2015) ${ }^{47}$. However, in a Brazilian study in patients with cardiovascular disease, it was found that the combination of ACE2 polymorphisms with those of ACE statistically significantly increases the risk of hypertension (ACE2 G allele and ACE DD genotype) ${ }^{48}$.

Another gene that could potentially affect the severity of COVID-19 is AGTR2, which encodes type 2 Angiotensin II receptors. The trials of this gene were carried out both under clinical conditions in patients with severe lung diseases and in experimental animal models. Genetic inactivation of AGTR2 by subcutaneous injection of a selective AGTR2 antagonist has been shown to improve lung function in cystic fibrosis. The role of AGTR2 in modeling insulin sensitivity and its allele-specific activity in muscle cells has been identified ${ }^{49}$. A biopsy study of patients with idiopathic pulmonary fibrosis showed that AGTR2-mediated effects of Angiotensin II dominate in activated myofibroblasts, which can lead to disruption of epithelial-mesenchymal interaction, thereby triggering fibrogenesis ${ }^{50}$. Thus, it can be assumed that it is the binding of 
SARS-CoV-2 to AGTR2 directly and (or) indirectly via the ACE2 receptor that leads to an imbalance in the RAS, a lack of Angiotensin (1-7) and excessive accumulation of Angiotensin II, and as a result to more severe forms of the disease ${ }^{51}$.

AGTR2 is indirectly involved in the regulation of NO synthetase (NOS) expression, which is also one of the main regulators of ventilation-perfusion relationships ${ }^{52,53}$. NOS3 is constantly expressed by the epithelium of the respiratory tract, the level increases with physical exertion, stress, chronic hypoxia. Nitric oxide is known as a strong endogenous agent that takes part in the processes of angiogenesis, immunoregulation, and the production of surfactants, which causes smooth muscle relaxation in blood vessels walls, increases the permeability of the endothelium and suppresses adhesion of platelets to the vessel wall. A decrease in the level of production of endogenous nitric oxide causes vasoconstriction, resulting in increased blood pressure.

The role of polymorphisms AGTR2 and NOS3 genes in the development of infectious processes and lung injury in newborns remains insufficiently examined. Two studies dealing with these genetic polymorphisms were performed in our Center and published in 2019. The first research work included 101 neonates with congenital pneumonia requiring respiratory therapy. The study showed the association of NOS3: -786 with severe manifestation of respiratory distress and the need for high frequency oscillatory ventilation (HFOV) in newborns with congenital pneumonia. Also, a statistically significant effect has been shown for a gene encoding type 1 AngII receptors (AGTR1). The C/C AGTR1 genotype was associated with a severe course of the disease requiring high parameters of ventilation ${ }^{54}$.

The second research was conducted in 379 newborns of gestational age (GA) 25-40 weeks with respiratory distress caused by infectious (early-onset neonatal sepsis or pneumonia) and noninfectious reasons (respiratory distress-syndrome and transient tachypnea, RDS, TTN) ${ }^{55}$. It was shown that the AGTR2 gene (its T allele) is associated with infectious disease in neonates. The total genotypic frequency in these age groups was 58\% for sepsis/pneumonia versus $36 \%$ for RDS/TTN ( $\mathrm{p}=0.00002$ ). Also a statistically significant increase in the frequency of carriage of alleles associated with a decreased NO level was revealed among neonates with infectious disorders (for NOS-786 T> C 62.5\% versus 34.6\%: OR = 3, 15 (1.21-8.17), p = 0.02). These studies showed that the development of early-onset infection and lung injury is associated with genes regulating vascular tone and energy metabolism, indicating a complex mechanism of the process. 
Thus, summarizing the results of genetic studies of the RAS, we can conclude that there is a complex interaction of elements (ACE2, AGTR2, AGTR1) both inside the system and with other pathways involved in the regulation of vascular tone, as well as cell growth and proliferation (NOS). A decrease in the activity of ACE2 triggered by the virus and an increase in the level of Ang-II, as a result, can have a damaging effect through various receptors and signaling molecules, which becomes more pronounced under the condition of an initial, genetically determined imbalance. In this regard, it is impossible to speculate about genetic predisposition to COVID-19 only based on ACE2 expression; all other genes studied to date (AGTR1, AGTR2, NOS) should also be taken into account.

\section{Conclusion}

It is now known that newborns and young children are less susceptible to COVID-19 and its severe forms. The explanation for this phenomenon may lie in the immunological characteristics in combination with the renin-angiotensin system features.

The protective properties of childhood immunity are immaturity of particular elements of the innate immune response, constitutional lymphocytosis with a shift in the subpopulation of lymphocytes towards anti-inflammatory Th2, as well as "trained" immunity. The cascade of RAS reactions is one of the key components in the pathogenesis of COVID-19, and in this review, it is considered from two perspectives: expression of ACE2 receptors and polymorphisms of certain genes of this system. It was established that the ACE2 transmembrane protein is not only the entry point for the virus, but also plays a regulatory role, turning the pro-inflammatory vasoconstrictor Angiotensin II into anti-inflammatory Angiotensin (1-7), which has vasodilating properties. Higher ACE2 content in children compared with adults helps maintain balance in the RAS and prevents the development of complications. It was also shown that the presence of certain genetic polymorphisms (AGTR1, AGTR2, ACE2, ACE) could determine the imbalance inside the RAS, leading to more pronounced reactions of alveolocytes, vascular endothelium and smooth muscle fibers in response to SARS-CoV-2 infection due to a shift towards vasoconstrictor, proliferative and profibrotic mechanisms.

The study of genetic polymorphisms is currently a promising method, which, together with immunological studies in COVID-19, contributes to a deep understanding of the essential elements in the disease pathogenesis and, based on this, could allow us to determine etiopathogenetically reasonable treatment approaches in patients of different age groups. 


\section{References}

1. Dong Y, Mo X, Hu Y, et al. Epidemiology of COVID-19 Among Children in China. Pediatrics. Published online March 16, 2020:e20200702. doi:10.1542/peds.2020-0702

2. Novel Coronavirus Pneumonia Emergency Response Epidemiology Team. [The epidemiological characteristics of an outbreak of 2019 novel coronavirus diseases (COVID19) in China]. Zhonghua Liu Xing Bing Xue Za Zhi Zhonghua Liuxingbingxue Zazhi. 2020;41(2):145-151. doi:10.3760/cma.j.issn.0254-6450.2020.02.003

3. CDCMMWR. Coronavirus Disease 2019 in Children — United States, February 12-April 2, 2020. MMWR Morb Mortal Wkly Rep. 2020;69. doi:10.15585/mmwr.mm6914e4

4. Grasselli G, Zangrillo A, Zanella A, et al. Baseline Characteristics and Outcomes of 1591 Patients Infected With SARS-CoV-2 Admitted to ICUs of the Lombardy Region, Italy. JAMA. Published online April 6, 2020. doi:10.1001/jama.2020.5394

5. Coronado Munoz A, Nawaratne U, McMann D, Ellsworth M, Meliones J, Boukas K. LateOnset Neonatal Sepsis in a Patient with Covid-19. N Engl J Med. 2020;0(0):e49. doi:10.1056/NEJMc2010614

6. Díaz CA, Maestro ML, Pumarega MTM, Antón BF, Alonso CP. First case of neonatal infection due to COVID 19 in Spain. An Pediatría Engl Ed.

doi:10.1016/j.anpede.2020.03.002

7. Zeng L, Xia S, Yuan W, et al. Neonatal Early-Onset Infection With SARS-CoV-2 in 33 Neonates Born to Mothers With COVID-19 in Wuhan, China. JAMA Pediatr. Published online March 26, 2020. doi:10.1001/jamapediatrics.2020.0878

8. Balduzzi A, Brivio E, Rovelli A, et al. Lessons After the Early Management of the COVID19 Outbreak in a Pediatric Transplant and Hemato-Oncology Center Embedded within a COVID-19 Dedicated Hospital in Lombardia, Italy. Estote Parati. (Be Ready.). Social Science Research Network; 2020. doi:10.2139/ssrn.3559560

9. Cristiani L, Mancino E, Matera L, et al. Will children reveal their secret? The coronavirus dilemma. Eur Respir J. Published online January 1, 2020. doi:10.1183/13993003.007492020

10. Darrah RJ, Jacono FJ, Joshi N, et al. AGTR2 absence or antagonism prevents cystic fibrosis pulmonary manifestations. J Cyst Fibros Off J Eur Cyst Fibros Soc. 2019;18(1):127-134. doi:10.1016/j.jcf.2018.05.013

11. Wat D. The common cold: a review of the literature. Eur J Intern Med. 2004;15(2):79-88. doi:10.1016/j.ejim.2004.01.006

12. Byrd-Leotis L, Cummings RD, Steinhauer DA. The Interplay between the Host Receptor and Influenza Virus Hemagglutinin and Neuraminidase. Int J Mol Sci. 2017;18(7). doi:10.3390/ijms18071541 
13. Yan R, Zhang Y, Li Y, Xia L, Guo Y, Zhou Q. Structural basis for the recognition of SARS-CoV-2 by full-length human ACE2. Science. 2020;367(6485):1444-1448. doi:10.1126/science.abb2762

14. Shulla A, Heald-Sargent T, Subramanya G, Zhao J, Perlman S, Gallagher T. A Transmembrane Serine Protease Is Linked to the Severe Acute Respiratory Syndrome Coronavirus Receptor and Activates Virus Entry. J Virol. 2011;85(2):873-882. doi:10.1128/JVI.02062-10

15. Wang K, Chen W, Zhou Y-S, et al. SARS-CoV-2 invades host cells via a novel route: CD147-spike protein. bioRxiv. Published online March 14, 2020:2020.03.14.988345. doi:10.1101/2020.03.14.988345

16. Newton AH, Cardani A, Braciale TJ. The host immune response in respiratory virus infection: balancing virus clearance and immunopathology. Semin Immunopathol. 2016;38(4):471-482. doi:10.1007/s00281-016-0558-0

17. Hanff TC, Harhay MO, Brown TS, Cohen JB, Mohareb AM. Is There an Association Between COVID-19 Mortality and the Renin-Angiotensin System - a Call for Epidemiologic Investigations. Clin Infect Dis. doi:10.1093/cid/ciaa329

18. Ahmadpoor P, Rostaing L. Why the immune system fails to mount an adaptive immune response to a COVID-19 infection. Transpl Int. n/a(n/a). doi:10.1111/tri.13611

19. Risitano AM, Mastellos DC, Huber-Lang M, et al. Complement as a target in COVID-19? Nat Rev Immunol. Published online April 23, 2020:1-2. doi:10.1038/s41577-020-0320-7

20. Senchenkova EY, Russell J, Almeida-Paula LD, Harding JW, Granger DN. Angiotensin IImediated microvascular thrombosis. Hypertension. 2010;56(6):1089-1095. doi:10.1161/HYPERTENSIONAHA.110.158220

21. Hedrich CM. COVID-19 - Considerations for the paediatric rheumatologist. Clin Immunol. 2020;214:108420. doi:10.1016/j.clim.2020.108420

22. Favalli EG, Ingegnoli F, De Lucia O, Cincinelli G, Cimaz R, Caporali R. COVID-19 infection and rheumatoid arthritis: Faraway, so close! Autoimmun Rev. 2020;19(5):102523. doi:10.1016/j.autrev.2020.102523

23. Mehta P, McAuley DF, Brown M, Sanchez E, Tattersall RS, Manson JJ. COVID-19: consider cytokine storm syndromes and immunosuppression. The Lancet. 2020;395(10229):1033-1034. doi:10.1016/S0140-6736(20)30628-0

24. Grom AA. Primary Hemophagocytic Lymphohistiocytosis and Macrophage Activation Syndrome: The Importance of Timely Clinical Differentiation. J Pediatr. 2017;189:1921.e1. doi:10.1016/j.jpeds.2017.07.025

25. Румянцев А.Г., Масчан А.А., Новичкова Г.А., Масчан М.А. ФЕДЕРАЛЬНЫЕ КЛИНИЧЕСКИЕ РЕКОМЕНДАЦИИ ПО ДИАГНОСТИКЕ И ЛЕЧЕНИЮ ГЕМОФАГОЦИТАРНОГО ЛИМФОГИСТИОЦИТОЗА. Published online 2016. 
26. Robert Kliegman Joseph St. Geme. Nelson Textbook of Pediatrics, 2-Volume Set 21st Edition; 2019.

27. McAdams RM, Juul SE. The role of cytokines and inflammatory cells in perinatal brain injury. Neurol Res Int. 2012;2012:561494. doi:10.1155/2012/561494

28. Wynn JL, Levy O. Role of Innate Host Defenses in Susceptibility to Early Onset Neonatal Sepsis. Clin Perinatol. 2010;37(2):307-337. doi:10.1016/j.clp.2010.04.001

29. Nikitina Irina V., Zhukova A.S., Vanko L.V., et al. Cytokine status of preterm newborns with infectious and noninfectious diseases.

30. Valiathan R, Ashman M, Asthana D. Effects of Ageing on the Immune System: Infants to Elderly. Scand J Immunol. 2016;83(4):255-266. doi:10.1111/sji.12413

31. Zhukova A.S., Nikitina Irina V., Vanko L.V., et al. Production of reactive oxygen species by peripheral blood phagocytes in preterm infants in the early neonatal period. 2:89-96.

32. Belyaeva A.S., Balashova E.N., Vanko L.V., Matveeva N.K., Milaya O.V., Krechetova L.V., Ionov O.V., Zubkov V.V., Degtyarev D.N. The phenotypic and functional characteristics of phagocytes in the blood of premature infants in the early neonatal period. Obstetrics and Gynecology. 2014;10:59-65.

33. Melville JM, Moss TJM. The immune consequences of preterm birth. Front Neurosci. 2013;7:79. doi:10.3389/fnins.2013.00079

34. Netea MG, Domínguez-Andrés J, Barreiro LB, et al. Defining trained immunity and its role in health and disease. Nat Rev Immunol. Published online March 4, 2020:1-14.

doi:10.1038/s41577-020-0285-6

35. Dolgikh S. Further Evidence of a Possible Correlation Between the Severity of Covid-19 and BCG Immunization. medRxiv. Published online April 10, 2020:2020.04.07.20056994. doi:10.1101/2020.04.07.20056994

36. Miller A, Reandelar MJ, Fasciglione K, Roumenova V, Li Y, Otazu GH. Correlation between Universal BCG Vaccination Policy and Reduced Morbidity and Mortality for COVID-19: An Epidemiological Study. Epidemiology; 2020. doi:10.1101/2020.03.24.20042937

37. Chamsi-Pasha MAR, Shao Z, Tang WHW. Angiotensin-Converting Enzyme 2 as a Therapeutic Target for Heart Failure. Curr Heart Fail Rep. 2014;11(1):58-63. doi:10.1007/s11897-013-0178-0

38. Jerng J-S, Hsu Y-C, Wu H-D, et al. Role of the renin-angiotensin system in ventilatorinduced lung injury: an in vivo study in a rat model. Thorax. 2007;62(6):527-535. doi:10.1136/thx.2006.061945

39. Ratliff B, Sekulic M, Rodebaugh J, Solhaug MJ. Angiotensin II regulates nitric oxide synthase expression in afferent arterioles of the developing porcine kidney. Pediatr Res. 2010;68(1):29-34. doi:10.1203/PDR.0b013e3181e12770 
40. Wagenaar GTM, Sengers RMA, Laghmani EH, et al. Angiotensin II type 2 receptor ligand PD123319 attenuates hyperoxia-induced lung and heart injury at a low dose in newborn rats. Am J Physiol Lung Cell Mol Physiol. 2014;307(3):L261-272. doi:10.1152/ajplung.00345.2013

41. Walls AC, Park Y-J, Tortorici MA, Wall A, McGuire AT, Veesler D. Structure, Function, and Antigenicity of the SARS-CoV-2 Spike Glycoprotein. Cell. 2020;181(2):281-292.e6. doi:10.1016/j.cell.2020.02.058

42. Xie X, Xudong X, Chen J, et al. Age- and gender-related difference of ACE2 expression in rat lung. Life Sci. 2006;78(19):2166-2171. doi:10.1016/j.lfs.2005.09.038

43. Asperen RMW, Lutter R, Specht PA, et al. Acute respiratory distress syndrome leads to reduced ratio of ACE/ACE2 activities and is prevented by angiotensin-(1-7) or an angiotensin II receptor antagonist. J Pathol. 2011;225(4):618-627. doi:10.1002/path.2987

44. Kuba K, Imai Y, Rao S, et al. A crucial role of angiotensin converting enzyme 2 (ACE2) in SARS coronavirus-induced lung injury. Nat Med. 2005;11(8):875-879. doi:10.1038/nm1267

45. Chen J, Jiang Q, Xia X, et al. Individual Variation of the SARS-CoV2 Receptor ACE2 Gene Expression and Regulation. Published online March 12, 2020. Accessed April 29, 2020. https://www.preprints.org/manuscript/202003.0191/v1

46. Informe sobre la situación de COVID-19 en España: Informe COVID-19 n 20. 3 de abril de 2020. Published online April 3, 2020.

https://www.isciii.es/QueHacemos/Servicios/VigilanciaSaludPublicaRENAVE/Enfermedad esTransmisibles/Documents/INFORMES/Informes\%20COVID-

19/Informe\%20n\%C2\%BA\%2020.\%20Situaci\%C3\%B3n\%20de\%20COVID19\%20en\%20Espa\%C3\%B1a\%20a\%203\%20de\%20abril\%20de\%202020.pdf

47. Lopera E, Graaf A van der, Lanting P, et al. Lack of association between genetic variants at ACE2 and TMPRSS2 genes involved in SARS-CoV-2 infection and human quantitative phenotypes. medRxiv. Published online April 25, 2020:2020.04.22.20074963. doi:10.1101/2020.04.22.20074963

48. Pinheiro DS, Santos RS, Jardim PCBV, et al. The combination of ACE I/D and ACE2 G8790A polymorphisms revels susceptibility to hypertension: A genetic association study in Brazilian patients. PloS One. 2019;14(8):e0221248. doi:10.1371/journal.pone.0221248

49. Bonàs-Guarch S, Guindo-Martínez M, Miguel-Escalada I, et al. Re-analysis of public genetic data reveals a rare $\mathrm{X}$-chromosomal variant associated with type 2 diabetes. Nat Commun. 2018;9(1):1-14. doi:10.1038/s41467-017-02380-9

50. Königshoff M, Wilhelm A, Jahn A, et al. The angiotensin II receptor 2 is expressed and mediates angiotensin II signaling in lung fibrosis. Am J Respir Cell Mol Biol. 2007;37(6):640-650. doi:10.1165/rcmb.2006-0379TR

51. Cui Q, Cui C, Huang C, et al. AGTR2, One Possible Novel Key Gene for the Entry of 2019-nCoV into Human Cells. Published online February 14, 2020.

doi:10.20944/preprints202002.0194.v1 
52. Robillard S, Mercier C, Breton V, et al. Ablation of angiotensin type 2 receptor prevents endothelial nitric oxide synthase glutathionylation and nitration in ischaemic abductor muscle of diabetic mice. Diab Vasc Dis Res. 2020;17(1):1479164119883978.

doi:10.1177/1479164119883978

53. Stennett AK, Qiao X, Falone AE, Koledova VV, Khalil RA. Increased vascular angiotensin type 2 receptor expression and NOS-mediated mechanisms of vascular relaxation in pregnant rats. Am J Physiol-Heart Circ Physiol. 2009;296(3):H745-H755.

doi:10.1152/ajpheart.00861.2008

54. Ionov O.V., Donnikov A.E., Bezlepkina M.B., Nikitina I.V., Balashov E.N., Kirtbaya A.R., Kryuchko D.S., Baibarina E.N. Relationship between polymorphism in NOS3, AGTR1, TLR9, DRD4 genes and severity of congenital pneumonia in newborns. Akusherstvo $i$ Ginekologiya/Obstetrics and Gynecology. 2019;5:102-111. doi:https://dx.doi.org/10.18565/aig.2019.5.102-111

55. Nikitina I.V., Donnikov A.E., Krogh-Jensen O.A., Lenyushkina A.A., Bystritsky A.A., Kryuchko D.S., Ionov O.V., Zubkov V.V., Degtyarev D.N.,. Congenital infectionassociated genetic polymorphisms in children. Akusherstvo $i$ Ginekologiya/ Obstetrics and gynecology 2019; 11: 175-85 (In Russian). 2019;11:175-185.

doi:https://dx.doi.org/10.18565/aig.2019.11.175-185 